\title{
Testing RFoF Link for Transmitting HF-OTHR Signal Between Transmitter and Receiver Shelters
}

\author{
Pavle Petrović, Member, IEEE, Bojan Džolić, Member, IEEE, Nikola Lekić, Member, IEEE, \\ Nemanja Grbić, Member, IEEE, Ana Ćupurdija, Member, IEEE, \\ Vladimir Orlić, Senior Member, IEEE, and Miljko Erić
}

\begin{abstract}
HF-OTHR site contains two equipment shelters, up to $1 \mathrm{~km}$ away from each other, that need to be connected for transmitting a FMCW signal. This connection, if realized using a coaxial cable, has shown itself to be vulnerable to external electromagnetic discharges, which can damage the sensitive radar equipment. This paper explores and tests the possibility of using Radio-Frequency-over-Fiber (RFoF) link as an alternative to a coaxial cable for transmission of signal, in order to ensure the immunity from external sources of electromagnetic interference (EMI).
\end{abstract}

Keywords - Radio-Frequency-over-Fiber - RFoF, HighFrequency Over-the-Horizon Radar - HF-OTHR, Electromagnetic Interference.

\section{INTRODUCTION}

$\mathrm{H}$ IGH-Frequency Over-the-Horizon Radar (HF-OTHR) is an effective solution to maritime monitoring at distances up to 200 nautical miles $(370 \mathrm{~km})$ from the shore, [1]-[4]. Equipment of the HF-OTHR system is stored inside two shelters, located up to $1 \mathrm{~km}$ apart [3]. A radar signal is generated inside the shelter located near the receiving array ( $\mathrm{Rx}$ shelter) of the radar and needs to be transmitted to the shelter near the transmitting array (Tx shelter), where it is amplified and fed into antennas which radiate it towards the sea. In existing OTHR installation in the Gulf of Guinea this connection is realized using a $50 \Omega$ coaxial cable, called RxTx cable. This cable is grounded at multiple places, according to standard [5].

Paper received April 15, 2021; revised June 20, 2021; accepted June 27, 2021. Date of publication July 31, 2021. The associate editor coordinating the review of this manuscript and approving it for publication was Prof. Grozdan Petrović.

This paper is revised and expanded version of the paper presented at the 28th Telecommunications Forum TELFOR 2020 [14].

This research is financed by Vlatacom Institute of High Technologies, Serbia, under the project \#148 - Vlatacom HighFrequency Over-the-Horizon Radar Generation 2 (vHF - OTHR2) Development.

Pavle Petrović, Nemanja Grbić, Ana Ćupurdija and Miljko Erić are with the School of Electrical Engineering, Univ. of Belgrade, Bul. kralja Aleksandra 73, 11120 Belgrade, Serbia and Vlatacom Institute of High Technologies, Bul. Milutina Milankovića 5, 11070, Belgrade, Serbia (email: pavle.petrovic@vlatacom.com, nemanja.grbic@vlatacom.com, ana.cupurdija@vlatacom.com,miljko.eric@vlatacom.com).

Bojan Džolić, Vladimir Orlić and Nikola Lekić are with Vlatacom Institute of High Technologies, Bul. Milutina Milankovića 5, 11070 Belgrade, Serbia (e-mail: bojan.dzolic@vlatacom.com, vladimir.orlic@vlatacom.com, nikola.lekic@vlatacom.com).
During regular operation of the HF-OTHR systems installed in the Gulf of Guinea, it was noticed that system components connected to both sides of Rx-Tx coaxial cable occasionally suffered damage to their terminal circuits. Installation of protective circuits (attenuators, Surge protectors, Over-voltage protectors) protected the system components, but the protective circuits themselves continued to be damaged from time to time, requiring occasional visits to remote HF-OTHR sites which can be expensive at best, and dangerous at worst of times. Due to the location of malfunctions, it was concluded that the surge of electricity that damaged the system was induced inside the Rx-Tx cable.

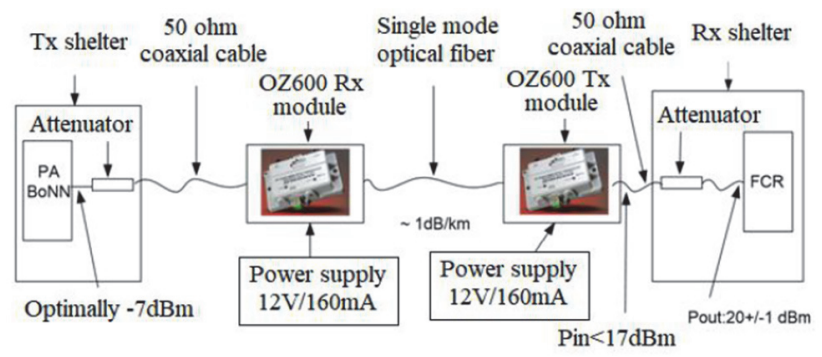

Fig. 1. Block schematic of shelters of HF-OTHR with a proposed realization of the RFoF link.

As a possible solution to this problem a RadioFrequency-over-Fiber (RFoF) link is proposed as an alternative to a coaxial cable for connecting $\mathrm{Rx}$ and $\mathrm{Tx}$ equipment shelters (Fig. 1). This link uses electro-optical converters to convert a radio frequency signal into an optical signal. This optical signal can then be transferred over optical fiber to its destination where it is converted back into a radio frequency electrical signal. This would galvanically isolate equipment shelters and critical components within: Frequency Control Rack (FCR) for radar signal generation and digital processing of received signals on Rx side and Power Amplifier (PA) on Tx side. As optical fibers are extremely resistant to electromagnetic interference (EMI) this would also solve the problem of external discharges damaging HF-OTHR equipment [6].

The approach towards the use of RFoF is elaborated in section II. Laboratory measurements of the RFoF link are presented in section III, while section IV contains the measurements done inside equipment shelters. Section V describes real in-the-field tests, while section VI presents the discussion of measurement results and a conclusion. 


\section{RADIO-FREQUENCY-OVER-FIBER LINK}

To combat these surges of electricity which can damage the equipment, the solution described in this paper was proposed. Instead of transmitting the signal from Rx to Tx shelters via the coaxial cable, the Radio-Frequency-overFiber link would be used. Various RFoF architectures can be found nowadays in practical use [7], including the ones implemented with commercial ICs [8] and those implemented in dedicated CMOS technology [9], [10] - for both fronthaul and downlink connection applications.

For this purpose, RFoF link was realized using a pair of OZ600 modules (Table 1), manufactured by Optical Zonu [11]. RFoF link consists of two separate modules, installed in $\mathrm{Rx}$ and Tx containers as shown in Fig. 2, connected with a single-mode optical fiber. To the best of the authors' knowledge, the RFoF link in question so far has not been tested for the extremely low-noise application such as HFOTHR.

TABLE 1: PARAMETERS OF INTEREST FOR OZ600 MODULES TAKEN FROM THE DATASHEET.

\begin{tabular}{l|r}
\hline Parameter & Value \\
\hline Gain & $3 \mathrm{~dB}$ \\
\hline Max. Input power & $17 \mathrm{dBm}$ \\
\hline Bandwidth & $10 \mathrm{kHz}-3.3 \mathrm{GHz}$ \\
\hline Phase characteristic & $\begin{array}{r}\text { Linear, group delay variations } \\
\text { listed for frequencies higher } \\
\text { than 30 MHz only. }\end{array}$ \\
\hline $\begin{array}{l}\text { Input/Output } \\
\text { Impedance }\end{array}$ & $50 \Omega$ \\
\hline Noise Figure & $40 \mathrm{~dB}$ \\
\hline
\end{tabular}

To be able to apply it as a connection between Rx and Tx shelters the following parameters had to be met:

- Gain/losses: Output signal must not drop below -7 $\mathrm{dBm}$;

- Phase characteristic must be linear;

- Noise figure: it must not introduce any excess noise compared to coaxial cable ( $>120 \mathrm{~dB}$ below signal);

- Phase noise: it must not raise phase noise of the signal compared to coaxial cable ( $>100 \mathrm{~dB}$ below signal).

Power budget for Rx-Tx link was calculated and given in Fig. 1. Additionally, Noise Figure is given in [12] to be $40 \mathrm{~dB}$. Maximal output power of FCR is $20 \mathrm{dBm}$. This power is attenuated by $10 \mathrm{~dB}$ before RFoF in order to protect the output of radar in case of malfunction of RFoF. This gives the theoretical limit of the signal to noise ratio (SNR) of around $90 \mathrm{~dB}$ for a signal with $100 \mathrm{kHz}$ bandwidth [13]. This SNR was deemed high enough for usage in HFOTHR. It is worth noting that this SNR is calculated for a situation when only thermal noise is present at the input of RFoF link. In real applications other sources of noise can also be present: in this implementation most dominant is quantization and phase noise of Digital-to-Analog converters in FCR. However, as these sources of noise exist regardless of what is used to connect $\mathrm{Rx}$ and $\mathrm{Tx}$ shelters, this analysis implies that realization of RFoF itself will not introduce significant noise into the system, which could degrade performance. After demodulation, FFT is performed on the signal which produces a system gain proportional to the duration of the sample, which improves SNR in the final result.

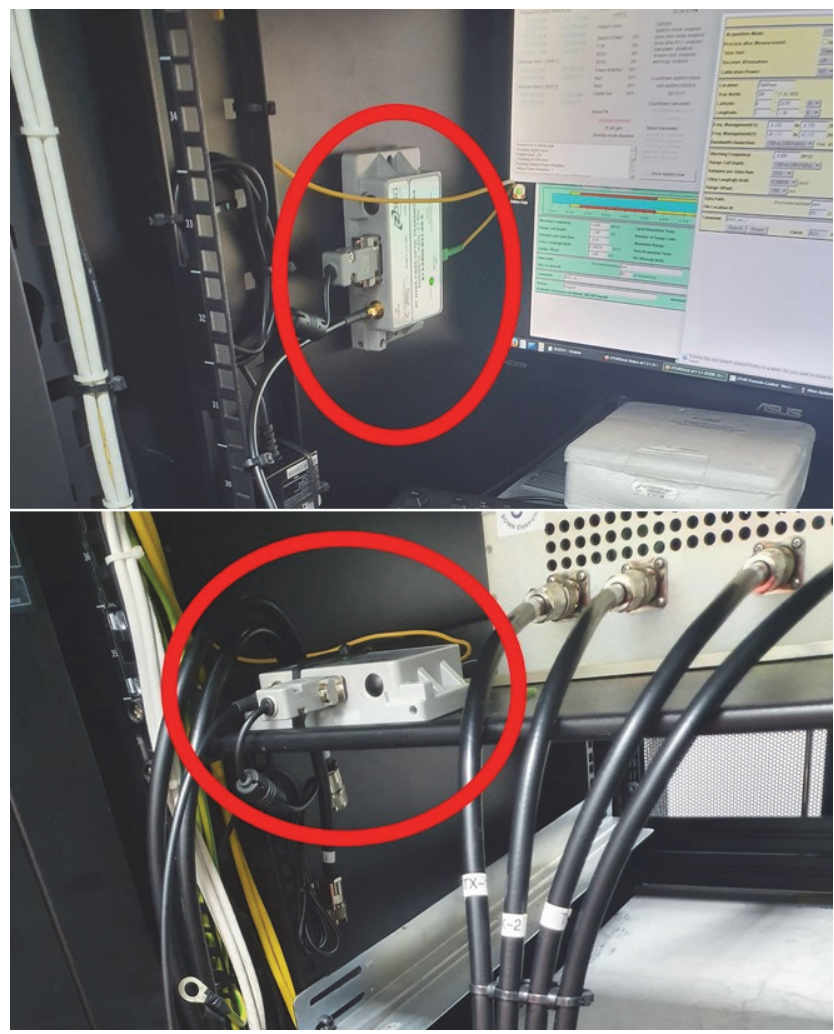

Fig. 2. RFoF transmitter, located in HF-OTHR Rx shelter (top) and RFoF receiver located in HF-OTHR Tx shelter (bottom).

\section{RFOF LABORATORY TESTING}

RFoF link had to be tested locally, in order to assure its proper operation before being integrated into existing systems, because any repair work after installation becomes progressively more difficult due to the remote location of HF-OTHR sites. A series of tests were performed on the RFoF link to quantify the parameters listed in the previous chapter. Firstly, the gain and the linearity of the phase were tested using the Vector Network Analyzer (VNA) Bode 100 , as shown in [14]. 2-port calibration was performed before the measurement at the ends of coaxial connectors. The frequency band at which the module was tested was between $4.4 \mathrm{MHz}$ and $5 \mathrm{MHz}$. The reason for that was because receiver modules used for later tests were optimized to operate in that band. The results showed a gain of around $5 \mathrm{~dB}$ in the band of interest, which is slightly higher than listed in the datasheet. Additionally, phase characteristic seemed to be linear.

Due to the way RFoF conversion is done, every $1 \mathrm{~dB}$ of losses in optical power corresponds to a $2 \mathrm{~dB}$ loss in $\mathrm{RF}$ power [12]. However, due to the low losses of modern optical fibers, it can be concluded that for distances of up to $1 \mathrm{~km}$, which are of interest, the RFoF link would still have a positive net gain [15].

Due to phase linearity, the group delay for the RFoF link is constant for the bandwidth of interest. To confirm this, measurement was done on a more sophisticated device, specifically ZND Rhode \& Schwarz Two-port network 
analyzer. The results confirmed the measurement of Gain, as shown in Fig. 3. Additionally, this new set of measurements showed a constant group delay value of around $258 \mathrm{~ns}$ from $4 \mathrm{MHz}$ to $11 \mathrm{MHz}$ when a $50 \mathrm{~m}$ optic fiber was used as shown in Fig. 4, which is consistent with earlier measurements and appropriate for usage in an $\mathrm{Rx}-$ Tx line without introducing unwanted signal distortion.

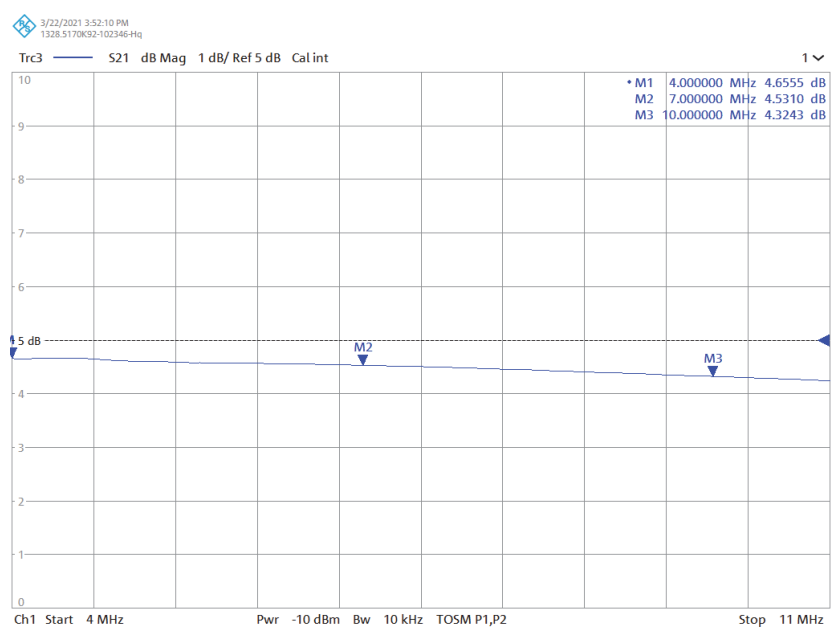

Fig. 3. Gain of RFoF link measurement from 4 to $11 \mathrm{MHz}$ measured on R\&S ZND Network Analyzer.

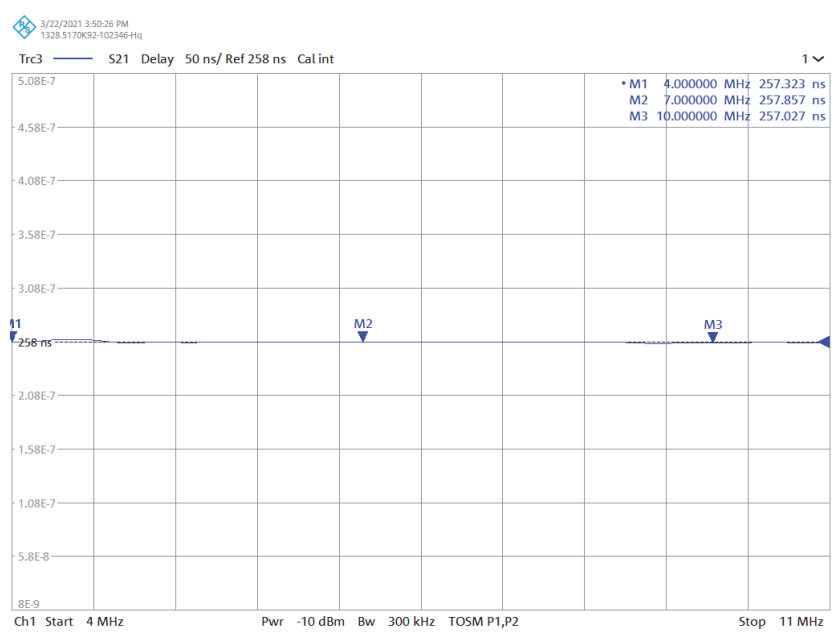

Fig. 4. Group delay measurement from 4 to $11 \mathrm{MHz}$ measured on R\&S ZND Network Analyzer.

Similar measurements can also be performed on-site to calculate the exact delay that the RFoF connection will introduce in the signal path. Then, HF-OTHR can be calibrated for this length, so as not to offset its range estimation due to delay. The same calibration is performed when the Rx-Tx cable is coaxial, so no additional changes to the system and signal processing need to be implemented. It is worth noting that optical fibers found commercially have a very low delay per unit length, so total delay of the signal is expected to be comparable to the delay in coaxial cable. All (declared and measured) properties of OZ600 modules and resulting RFoF link confirmed good potential for use in real applications.

\section{TESTING IN HF-OTHR SHELTERS}

Any excess noise inserted on the line between $\mathrm{Rx}$ and $\mathrm{Tx}$ shelters will be amplified by PA and radiated, which can severely impair the ability of the system to discern distant targets. For that reason, the amount of noise inserted with RFoF link between FCR and PA needs to be compared to the amount of noise existing when PA is connected to FCR with a coaxial cable. To measure the level of noise inserted by RFoF link, external sources of noise had to be excluded, because they would be picked up by receiving antennas regardless of whether a coaxial cable or RFoF is used. Additionally, the receiver of HF-OTHR has a much finer frequency resolution than any Spectrum Analyzers (SA) the authors had access to. This allows the mean noise level to be reduced sufficiently so that the phase noise levels can be gauged with great precision. It is for this reason that noise measurements were performed in the shelters of HF-OTHR, using its transmitter, Power amplifier, and receiver modules.

The measurement setup for comparing the amount of noise with and without the RFoF link is shown in Fig. 5. In the control setup the only difference was that instead of RFoF link, a coaxial cable was used. RER1 and RER2 are receiver racks, each of which contains eight receiver modules. Attenuators at both ends of connection between $\mathrm{Rx}$ and Tx serve a dual purpose of attenuating a signal to an optimal level for PA, and protecting equipment (FCR and PA) by providing a good impedance match for both of them in case a connection between them is removed for any reason. To test both a coaxial cable connection and RFoF link, a set of measurements with a sinusoidal excitation was performed.

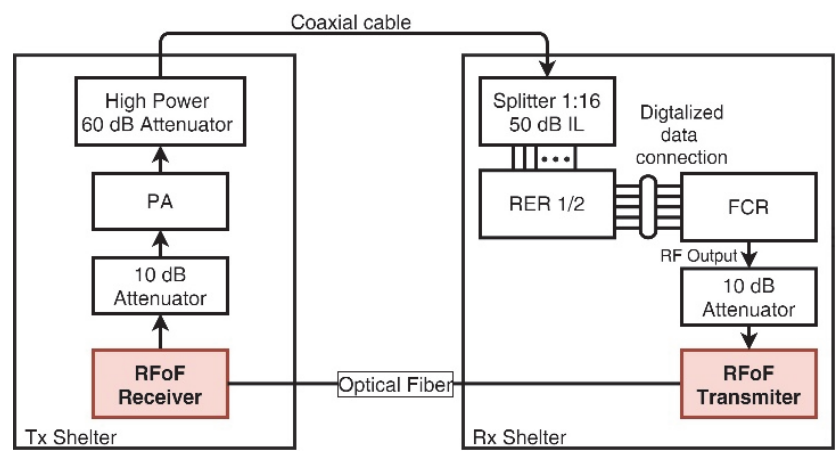

Fig. 5. Measurement setup for measuring noise and phase noise insertion of RFoF link.

Instead of a regular chirp signal, the FCR was set to emit a sinusoidal signal at the frequency of $4.6 \mathrm{MHz}$, for the predefined duration of around 67 seconds, which is equal to 256 chirp intervals. That signal is transmitted towards the PA, amplified, then attenuated to a sufficient level, and divided across the sixteen receiver channels of HF-OTHR. There, a signal is demodulated by a sinusoidal signal at a frequency that is $80 \mathrm{~Hz}$ away from the received signal. This, after IQ demodulation, leads to a demodulated signal in the form of a complex sinusoid at the frequency of $-80 \mathrm{~Hz}$. The demodulated signal is sampled with an Analog-to-Digital converter at the frequency of sampling of $V_{\text {sample }} \approx 6 \mathrm{kS} / \mathrm{s}$.

A Fast Fourier Transform (FFT) was performed on the resulting samples and a part of the spectrum between $280 \mathrm{~Hz}$ and $120 \mathrm{~Hz}$ was compared for a coaxial connection and RFoF connection. The frequency resolution of this FFT is $\Delta f=0.015022 \mathrm{~Hz}$, which is much finer than the resolution 
of most commercially available spectrum analyzers.

When it comes to noise levels and phase noise levels, HF-

OTHR requirements are extremely strict:

- Noise level after demodulation has to be at least $120 \mathrm{~dB}$ below the nominal level for a given frequency resolution;

- Phase noise after demodulation at $\pm 2 \mathrm{~Hz}$ from a demodulated sinusoidal signal has to be at least $100 \mathrm{~dB}$ lower than the signal.

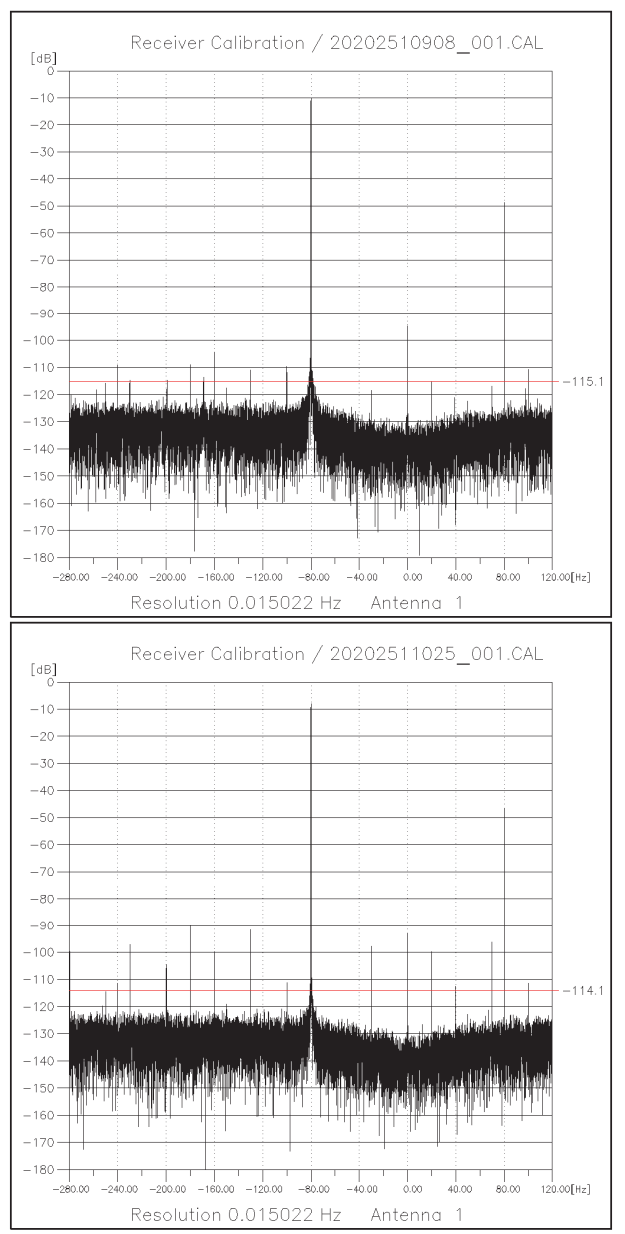

Fig. 6. Noise measurement when Tx and Rx are connected with a coaxial cable (top) and RFoF link (bottom).

Fig. 6 shows the results of this measurement, for receiver channel 1, when FCR and PA are connected using a coaxial cable as well as the results of the same measurement when a connection is realized via an RFoF link. Both of these figures show a spectrum of demodulated signal in a logarithmic scale, relative to a nominal receiver signal level of $-45 \mathrm{dBm}$.

Measurements with chirp signals were also performed. Measurement setup was the same, only instead of emitting a continous sinusoidal signal, FCR was set up so that it emits chirps of aproximate duration of $260 \mathrm{~ms}$. The time of measurement stays the same, at 67 seconds. Emitted and reference signals were again separated in frequency by 80 $\mathrm{Hz}$, which leads to a simulation of radar target at the $20^{\text {th }}$ resolution cell. On this set of chirps both the Range and Doppler processing algorithms were performed [1], [16]. Results of these measurements are shown in Fig. 7. From both Fig. 6 and Fig. 7 it can be concluded that strict requests in the quantitative level of noise figure and phase noise, as given above, are completely met.

\section{RFoF LinK MEASUREMENTS IN THE FIELD}

As RFoF link showed satisfying results in the laboratory setting, it finally qualified for installation within the operational HF-OTHR system [17]. The system chosen for installation is located in the region of West Africa, the Gulf of Guinea, where RFoF link installation has been performed in November 2020.
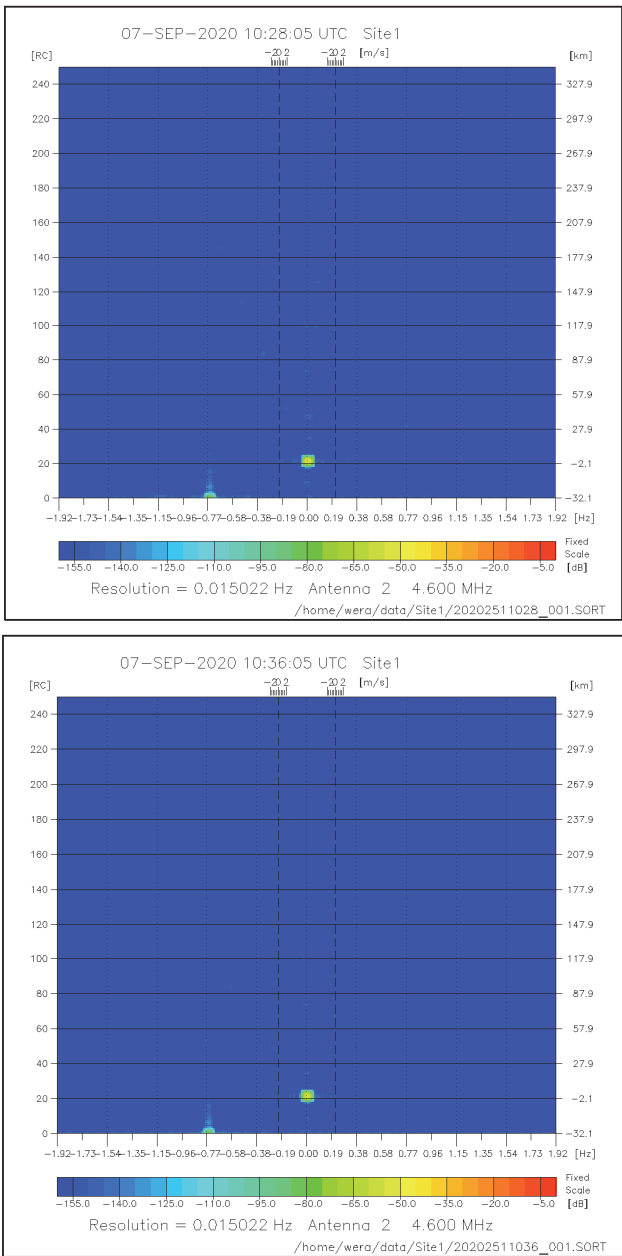

Fig. 7. Range-Doppler map of a simulated radar target when Rx-Tx connection is realized via coaxial cable (top) and via RFoF link (bottom).

The transmitter and receiver of RFoF were connected via already existing optical fiber between $\mathrm{Rx}$ and $\mathrm{Tx}$ shelter, as shown in Fig. 8. After installing an appropriate attenuation to adjust the signal level at the input of the PA, the radar was switched on and ran in regular operation. In the same way, as the link was tested in the laboratory [15], two sets of measurements were performed, using a sine signal and using an FMCW signal. The main difference from previously described tests was that now, instead of the signal being coupled over attenuator, the signal was emitted by a Tx antenna array, and echoes reflected from the environment were picked up using an $\mathrm{Rx}$ antenna array. For that reason, a higher level of external noise enters the receiver [18], as illustrated in achieved noise measurements in Fig. 9. Alongside that, reflections of radio waves in the HF band from the sea result in the characteristic Bragg 
scattering [1], which can be seen in Fig. 10 as two vertical lines of strong reflections in the Range-Doppler map.

Fig. 10 shows that there is significantly more noise in the system now, when compared to measurements in the laboratory, being a result of the presence of all the external noise, regularly pronounced in the High-Frequency band.

Bragg lines are seen in Fig. 10, which stretch for a significant portion of the range axis, correspond to signal reflected by Bragg scattering from sea waves at different ranges from the radar. As it can be seen in both Fig. 9 and Fig. 10, the results of corresponding measurements with a coaxial cable and an RFoF link practically do not differ at all in either the noise level or the range at which Bragg lines are present - representing the measure important in practice, as it is correlated to the maximum range of detection for the particular radar. Strict requests in the quantitative level of noise figure and phase noise are not completely met in this case, due to external noise that enters the system at the receiving antennas. However, it can be seen that the quantitive level of noise in both cases, using a coaxial cable and an RFoF link, are the same. This further shows that RFoF link does not introduce any excess noise. These results showed in the field that a coaxial cable could be replaced with an RFoF link in HF-OTHR system without any loss in performance.

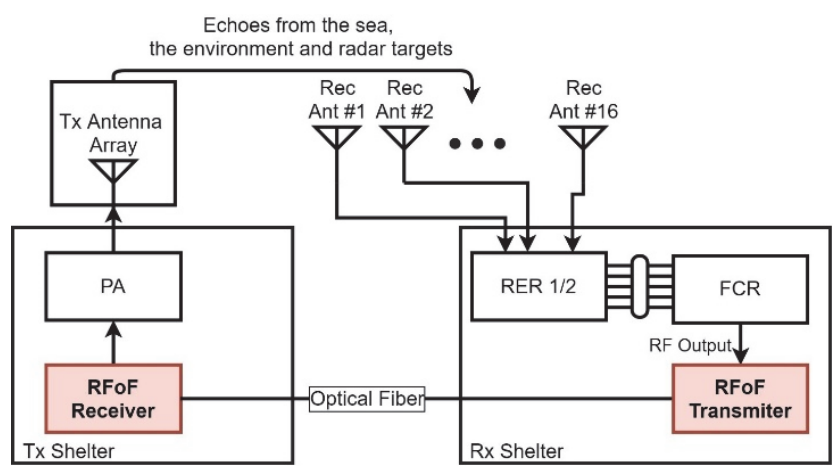

Fig. 8. Measurement setup in the field.

It is also worth noting that, during the 5 months since RFoF link has been installed in the field, no failures in the Rx-Tx line were reported. Several storms with lightning strikes that damaged other sensitive systems in the vicinity of HF-OTHR site happened during the same period, but the HF-OTHR system with RFoF link continued its operation completely unimpeded, causing no need for any maintenance activities. In this manner, the practical value of the solution presented in this paper has been additionally confirmed.

Analysis from the point of financial aspects shows that RFoF link implementation leads to approximately 3 times higher initial costs, in comparison with a traditional coaxial cable solution. Nevertheless, this financial cost is directly compensated through savings achieved in avoiding only one intervention for maintenance in the field, done by engaging human resources. According to experience in HFOTHR systems' employment, this initial investment in an RFoF link pays out in around one month of practical use. In future work, it would be of interest to explore the possible benefits of using RFoF solutions in other segments of HFOTHR system, such as antenna RF signal links, as an

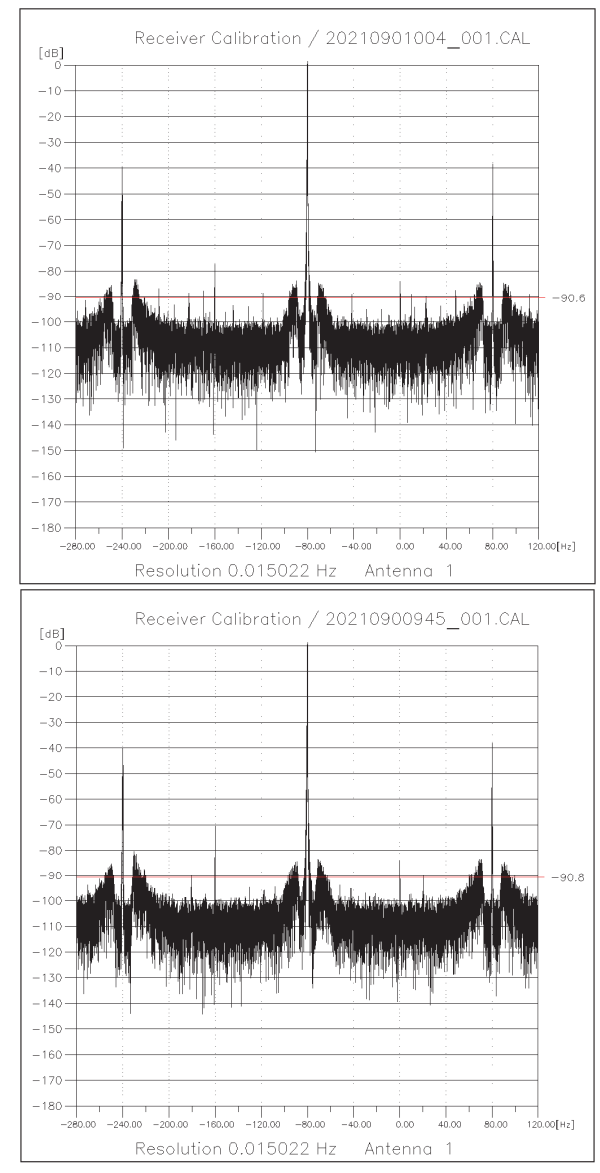

Fig. 9. Results of noise measurement after demodulation when coaxial cable is used (top) and when RFoF link is used (bottom).

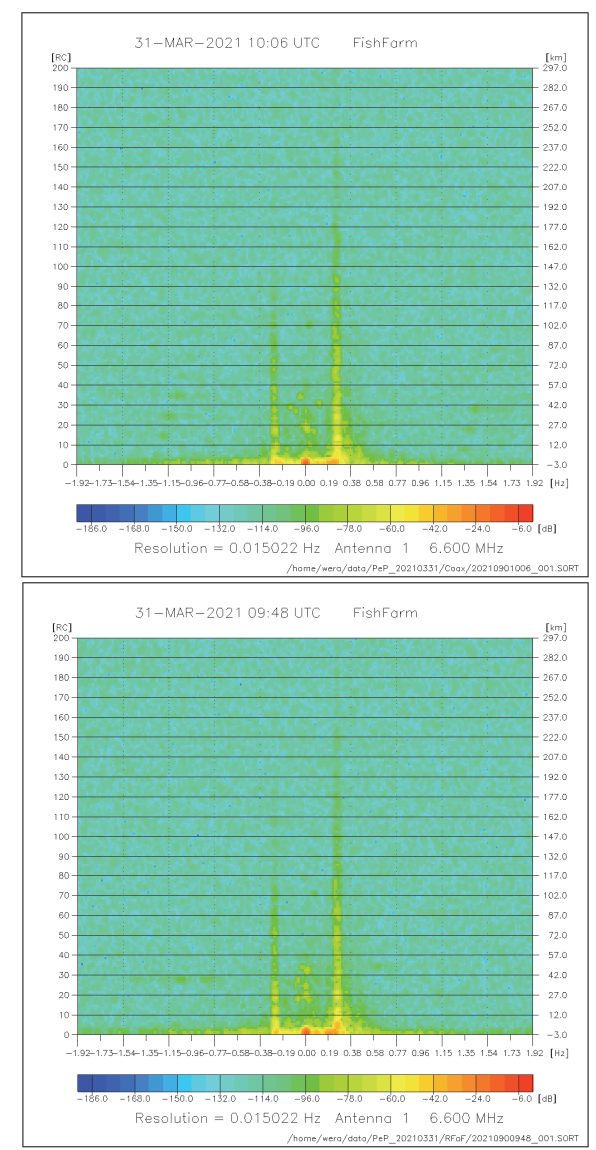

Fig. 10. Range-Doppler map acquired when the system is connected via coaxial cable (top) and RFoF link (bottom). 
example. While being also potentially vulnerable to external EMI, antenna links are extremely sensitive to noise performance, which should be treated with proper care in future considerations.

\section{CONCLUSION}

RFoF link was tested as a connection between Tx and Rx shelters of the HF-OTHR system. All of the requirements given in chapter III were fulfilled successfully. The gain of tested RFoF link is around $6 \mathrm{~dB}$, constant over the bandwidth of interest. It can be expected that this gain will drop with an increase of length of optical fiber, but it is not expected that this lowering of gain will influence the operation for ranges of interest. Phase characteristic was linear, which translates to a constant group delay over the entire bandwidth. These results are quite satisfactory.

There is no noticeable noise or phase noise increase when an RFoF link is used compared to the case where a coaxial cable is used. Test with a simulated radar target showed that an RFoF link transferred the chirp signal as well as the sinusoidal signal, with a simulated target as clearly defined as with a coaxial cable.

Results of these tests imply that an RFoF connection performs at least as well as a coaxial cable when it comes to connecting Rx and Tx shelters of HF-OTHR: it does not degrade radar processing. These results are promising and are an incentive for the RFoF links to be installed in already existing HF-OTHR systems. Thus, further testing has been done with RFoF modules installed in operating HF-OTHR systems in the Gulf of Guinea. The true test of RFoF link, i.e., its ability to protect the system from external EMI, was performed in the field, during regular radar operation.

The final in-the-field testing lasted for 5 months and showed no difference in performance between the standard coaxial cable and RFoF connection, while still being able to endure rigorous external conditions, much more challenging than the ones present in the laboratory. Additionally, the high reliability of the RFoF link solution was confirmed in practice, under true real-world conditions, providing no failures in the $\mathrm{Rx}$ - Tx line during the whole test period, even under several storms with lightning strikes, which damaged many other sensitive systems in the same area. For these reasons, the described RFoF link solution has been formally promoted into an integral part of the new generation of Vlatacom's HF-OTHR systems.

\section{REFERENCES}

[1] G. A. Fabrizio, High Frequency Over-The-Horizon Radar, 1st ed. New York City, USA: McGraw-Hill Education, 2013.

[2] D. Nikolic, B. Dzolic, N. Tošic, N. Lekic, V. D. Orlic, B. M. Todorovic: "HFSW Radar Design: Tactical, Technological and Enviromental Challenges," Proc. OTEH 2016, Belgrade, Serbia, pp. 349-354, 6.-7. October 2016.

[3] D. Nikolic, N. Tosic, B. Dzolic, N. Grbic, P. Petrovic, A. Djurdjevic, N. Lekic: "Tailoring OTHR Deployment in Order to Meet Conditions in Remote Equatorial Areas," Proc. CSPA 2019, Penang, Malaysia, 8.-9. March 2019.

[4] N. Stojkovic, D. Nikolic, B. Dzolic, N. Tosic, V. Orlic, N. Lekic, B M. Todorovic: "An Implementation of Tracking Algorithm for OverThe-Horizon Surface Wave Radar," Proc. TELFOR 2016, Belgrade, Serbia, 22.-23. November 2016., DOI: 10.1109/telfor.2016.7818820

[5] TIA-Telecommunications Industry Association, "TIA-607-B Generic Telecommunications Bonding and Grounding (Earthing) for Customer Premises." (2011).

[6] V. Everett, K. T. Jones, G. B. Scelsi, G. A. Woosley: "Measurement of Electrical Discharge Parameters Using Optical Fibres," Australian Journal of Physics, vol. 48, iss. 3, p.527.

[7] D. F. Paredes-Páliz, G. Royo, F. Aznar, C. Aldea, and S. Celma, "Radio over fiber: an alternative broadband network technology for IoT," Electronics, vol. 9, no. 11, (2020): 1785.

[8] M. Sung, S. Cho, J. Kim, J. K. Lee, J. H. Lee and H. S. Chung, "Demonstration of IFoF-Based Mobile Fronthaul in 5G Prototype With 28-GHz Millimeter wave," Journal of Lightwave Technology, vol. 36 , no. 2, pp. 601-609, 15 Jan. 15, 2018

[9] W. Ahmad, M. Abdulaziz, A. Nejdel, M. Törmänen and H. Sjöland, "CMOS Integrated Remote Antenna Unit for Fiber-Fed Distributed MIMO Systems," IEEE Transactions on Microwave Theory and Techniques, vol. 65, no. 1, pp. 173-186, Jan. 2017.

[10] G. Royo, A. D. Martinez-Perez, C. Sanchez-Azqueta, C. Aldea, and S. Celma, "A highly linear low-noise transimpedance amplifier for indoor fiber-wireless remote antenna units," Electronics, vol. 8, no. 4, (2019): 437.

[11] OZ600 datasheet. Available: https://www.opticalzonu.com/

[12] RF Over Fiber Design Guide Overview. Available: https://www.opticalzonu.com/

[13] M. Eric, B. Dzolic, N. Lekic, N. Tosic, P. Petrovic, and N. Dumbelovic: "Elaboration of connecting shelters of vHF-OTHR system using RFoF link," Internal document, Vlatacom Institute of High Technologies, 2019.

[14] P. Petrović, B. Džolić, N. Lekić, N. Grbić, A. Ćupurdija, V. Orlić, and M. Erić, "Testing RFoF Link for Transmitting HF-OTHR Signal Between Transmitter and Receiver shelters," 2020 28th Telecommunications Forum (TELFOR), pp. 1-4. IEEE, 2020.

[15] M. Eric, N. Lekic, N. Tosic, N. Dumbelovic, B.Dzolic, P. Petrovic, and N. Grbic: "RFoF link laboratory testing report", Internal document, Vlatacom Institute of High Technologies, 2020.

[16] M. Skolnik, Radar Handbook 2nd edition, McGraw-Hill, inc. 1990.

[17] D. Nikolić, N. Stojković, P. Petrović, N. Tošić, N. Lekić, Z Stanković, and Nebojša Dončov, "The high frequency surface wave radar solution for vessel tracking beyond the horizon," Facta universitatis - series: Electronics and Energetics, vol. 33, no. 1, pp. 37-59, 2020.

[18] Spaulding, Arthur D., and James S. Washburn, Atmospheric radio noise: Worldwide levels and other characteristics. US Department of Commerce. National Telecommunications and Information Administration, 1985. 